\title{
MIMO-WSN implementation in geriatric care unit through MASTS and successive convex approximation algorithm.
}

\author{
Duraichi $\mathbf{N}^{1,2^{*}}$, Suganthi $\mathbf{M}^{3}$ \\ ${ }^{1}$ Research Scholar, Anna University, Chennai \\ ${ }^{2}$ Veltech Hightech Dr. Rangarajan Dr. Sakunthala Engineering College, Tamil Nadu, India \\ ${ }^{3}$ Department of Electronics and Communication, Thiagarajar College of Engineering, Tamil Nadu, India
}

\begin{abstract}
Base on the current situation of the development of wireless medical, according to the multi-frequency and broadband MIMO road medical special wireless network system themselves security, stability and continuity of network transmission, wireless network impact on human health and the medical equipment is discussed. Aging is associated with a decrease in the physiologic reserve thus; many elderly persons are unable to compensate for the increased metabolic demands that accompany acute illness or injury. This inability to compensate leads to increased rates of postoperative complications and death. Aggressive, goal-directed management in the Geriatric intensive care unit is beneficial for the geriatric patient. In this paper MIMO WSN is used in patient monitoring scenario to collect the bio-physiological variables and to trigger emergency services. MASTS algorithm is implemented for channel selection and SCA algorithm is for energy maximization throughput. The performance measure like end to end delay, packet delivery ratio and throughput are analysed through simulation and graphs are shown.
\end{abstract}

Keywords: Geriatric care unit, MIMO, WSN, Network life, SCA.

Accepted on May 22, 2017

\section{Introduction}

Several network architectures and wireless technologies that can be used for the implementations of WSN in patient monitoring schemes. Now MIMOWSN deployed in patient monitoring scenario and the patient pathology it may be necessary to monitor different bio-physiological variables. The collected data can be used for remote diagnosis, consolatory patient monitoring and to trigger emergency services, the current progress in the field of wireless technology allows developing small size sensors called nodes, communicating with each other via a radio link and are characterized by their limited resources (power supply, processing, memory storage). Their flexibility of use makes them more utilized to form a Wireless Sensor Network (WSN) without returning to a fixed infrastructure. Furthermore, these nodes typically deployed in inaccessible areas to control a definite phenomenon, ensure the transfer of data collected using a multi-hop routing to a Base Station (BS), which is far from the monitored field. The BS is responsible for the analysis and processing of collected data to be exploited by the end user. Based on the above briefly description, for a WSN to accomplish its function without failure of the connection between the nodes, because of a depleting battery of one or more nodes, we need a data routing protocol which gives higher priority to the energy factor compared to other limitations, in order to provide stability of

the network. At this point, many studies have been made; the most known ones are based on the MIMO technologies. However, the node cannot carry multiple antennas at the same time due to its limited physical size. Therefore, a new transmission technique called Cooperative MIMO" has been proposed. This technique is based on the cooperation principle where the existence of different nodes in the network is exploited to transmit the information from the source to a specific destination by virtually using the MIMO system. The Cooperative MIMO allows obtaining the space-time diversity gain, the reduction of energy consumption, and the enhancement of the system capacity.

MIMO is used in conjunction with spatial multiplexing as a way to increase the peak data rate. This method uses additional transmit and receive antennas to transmit parallel data streams. As a result, the channel capacity is increased without increasing the bandwidth or the SNR. With an Msx MrMIMO constellation-where Msis the number of transmit antennas and Mris the number of receive antennas-the maximum number of spatially separate data streams is defined as $\mathrm{M}=\min (\mathrm{Ms}, \mathrm{Mr}$ ). A MIMO system consists of several antenna elements, plus adaptive signal processing, at both transmitter and receiver. 


\section{Related Work}

Remote sensing of heart and respiration activity by measuring Doppler shift in radio signals is detected using MIMO techniques. Based on BLAST to isolate useful scattered radio signals from those due to unwanted motion in the environment. If there is interfering motion in the radar field of view, multiple antennas can be used to detect multiple copies of the same signal with different phase information, with coherent combining used to provide a greatly improved estimate of desired Doppler motion. MIMO system indicates that it is possible to separate multiple subjects even with identical breathing and heart rates. To numerically assess the performance of the MIMO radar sensing system, model designed as a human body as a cluster of isotropic scatters. The scatterers are randomly distributed around the cluster center, within a circle of diameter D. The cluster contains Ns scatterers. Simulations were done for a MIMO system with 4 transmit and 4 receive antennas, with the carrier frequency of $2.4 \mathrm{GHz}$, and $2 \lambda$ and $\lambda / 2$ separation between transmit and receive antenna elements [1].

In vivo channel, achieving high bit rate approximately 100 Mbps performance will be facilitated by the use of MIMO Communication. In vivo Capacity, The OFDM system can be modelled as $Y_{\mathrm{k}}=H_{\mathrm{k}} X k+W k K=1,2 \ldots \ldots . N$ data [2].

Multiple-Input Multiple-Output (MIMO) systems employing a spatial array of antennas that enhance the channel capacity. Systems with three- and four-element vector antennas at both the transmitter and receiver operating around the frequency of $2.25 \mathrm{GHz}$ support three and four times more information. Simplified theoretical model of a MIMO system with colocated antennas in a rich multipath environment shows good agreement. The properties of two such MIMO systems are investigated. The first employs a vector antenna consisting of three elements, namely, a loop and two coplanar dipoles, and operates at $2.22 \mathrm{GHz}$. The second system consists of a fourelement vector antenna including a loop and three orthogonal dipoles and operates at $2.27 \mathrm{GHz}$. The properties of a MIMO communication link employing these two vector antennas are investigated experimentally and the increase in Expected Mutual Information (EMI) is observed in a realistic scattering environment. The favorable characteristics of these systems in being as effective as traditional spatial antenna arrays within the physical space of a single antenna could lead to their use in a number of applications, including high data-rate communication links, angle of arrival estimation, and sensors, to name a few [3]. A new technique for Multiple-Input Multiple-Output (MIMO) radar with colocated antennas which we call phased-MIMO radar. MIMO radar techniques are demonstrated analytically and by simulations through analyzing the corresponding beam patterns and the achievable output signal-to-noise-plus-interference ratios. MIMO radars can be classified into two main types. The first type uses widely separated transmit/receive antennas to capture the spatial diversity. In this case, the waveform diversity is similar to the multi-path diversity concept in wireless communications over fading channels. The second MIMO radar type employs arrays of closely spaced transmit/receive antennas to cohere a beam towards a certain direction in space. This technique is based on partitioning the transmit array to a number of sub arrays which are allowed to overlap. Each sub array is used to coherently transmit a waveform which is orthogonal to the waveforms transmitted by other sub arrays. The transmit beam forming and transmit sub array waveform designs, which satisfy certain desired properties [4]. Methodology named channel selection with the aim of balancing the MIMO advantage and the complexity of sensor co operations. MASTS algorithm is to compute a spanning tree with the maximum sum of weight of edge, i.e., to select the maximum sum of channel gain while realizing the connectivity of all the sensors on a basis of maximum spanning tree algorithm. To apply the graph theoretical concept on maximum spanning tree into virtual MIMO channel selection. In order to increase the data rate, different transmitter simultaneously transmits different symbols. MASTS virtual MIMO can achieve even better capacity with/without water-filling, less diversity gain and similar multiplexing gain as those of full virtual MIMO [5]. To reduce the energy used by sensor nodes the cooperative MIMO and data aggregation techniques are combined. A new energy model that considers the relationship between data generated by nodes and the distance between them for a cluster-based sensor network by employing the combined techniques. Energy and link Efficient Clustering Technique for reliable routing (ELECT) considers current status of node, link condition and uses a clustering metric, called Predicted Transmission Count (PTX) and gateway. Using various spectral clustering techniques an algorithm proposed, called Spectral Classification for Robust Clustering in Wireless Sensor Networks (SCRC-WSN) [6]. Here in this spectral partitioning method, graph theory is used partition the fixed network into clusters. All the nodes that form cluster send data to cluster head which is elected by them and cluster head further send data to base station. The different steps of the SCRC-WSN protocol are: 1. preprocessing, 2. clustering, 3. cluster head election, 4. steady state phase. CMIMO technique which is based on clustered random wireless network just like the LEACH protocol. The operation of CMIMO has three main phases: cluster formation, intra cluster communication, inters cluster communication. Designing of CMIMO includes listening cost, synchronization, reclustering and MAC. In CMIMO multiple nodes placed adjacently with single antenna form a virtual antenna array to achieve spatial diversity. CMIMO adapts transmission modes and transmission power on per packet basis [7]. WWAN is a long-range communication that allows doctors and caregivers to monitor the patient's data by using cellular network data anywhere and also with the utilization of internet. WWANs establish connection over large areas, like cities or countries, via multiple satellite systems or antenna sites looked after by an Internet Service Provider (ISP). The types of wireless networks such as WPANs, WLANs, WMANs, and WWANs which can be utilized efficiently for health care monitoring systems in WSNs. By choosing the appropriate wireless network type for the specific application, deployment cost can be minimized and also challenges related to wireless data transmission are also 
reduced [8]. An application of Multiple-Input Multiple-Output (MIMO) radar techniques to medical imaging is investigated that MIMO radar, which can be viewed as a form of microwave tomography for medical imaging geometries, offers an alternative to current X-ray technology. A signal processing approach has been discussed for microwave detection of tumors in breast tissues. By combining multiple mitigation approaches, self-interference is reduced, despite the effects of calibration errors. After mitigation, accurate tumor size estimates are analysed [9]. The optimal assignment of each mobile user to a cloud server through the most convenient base station and, contextually, the optimal MIMO precoding matrices and computational rates (virtual machines) to each user, a network composed of a set of $N c$ clouds, $N b$ small cell access points and $\mathrm{K}$ mobile user equipment's (MUEs). Each access point $\mathrm{n}$ is connected to the cloud provider $\mathrm{m}$ through a backhaul link assumed to be a high speed connection. MIMO precoding and computational rates seem to provide a powerful mechanism to handle computation offloading. The proposed approach gives also a strategy to handle hand-over, in case of low mobility, taking into account not only the wireless traffic in each base station, but also the computational load and the state of the backhaul connecting different clouds [10]. The new infrastructure includes multi-purpose nodes that run a Network Operating System (NOS). Joint beam forming with Virtual Cell approach proved its ability to increase the system capacity, while cloud computing and virtualization outrun the experimental stages and are ready to be applied on the new Clod Networking [11]. Large Scale Antenna array (LSA) can be also combined with OFDM to deal with frequency selectivity in wireless channels. However, such combination suffers from substantially increased complexity. First, the precoding is conducted in the frequency domain for traditional MIMO-OFDM [9]. In this case, each antenna at the BS requires an Inverse Fast Fourier transform (IFFT) for OFDM modulation and the number of IFFTs is equal to the antenna number. Second, Zero-Forcing (ZF) precoding is required to support more users in LSA systems. It is more suitable to adopt the convolutional precoding rather than the traditional frequency-domain precoding for the transceiver design in LSA-OFDM systems [12]. Multiple MIMO full-duplex relays are deployed in a network, the signal reception quality relies on the effective suppression of multiple types of interference. The distributed beam forming is studied for the MIMO FDR network, by formulating a power minimization problem with a non-strict convex objective function. SLIPD-based distributed beam forming algorithm for $\mathrm{Nr}=1$ for the case of single receive antenna at relays and SLIPD-based distributed beam forming algorithm for $\mathrm{Nr}>1$ for the case of multiple receive antennas at relays). A globally optimal solution of the power minimization problem can be obtained by Algorithm 1, while Algorithm 2, can only yield a local optimal solution, due to conservative SCA performed at each iteration [13]. A switching criterion that chooses the transmission mode that maximizes the DL total channel capacity while keeping the UL channel capacity at a certain level. Additionally, the switching criterion was formulated as a resource allocation problem and then solved using the KKT conditions. Novel multiuser beam forming algorithms for user fairness and sum rate maximization, utilizing newly derived convex optimization problems for transmit and receive beam formers calculation in a recursive optimization [14]. Both beam forming algorithms provide efficient operation for the interference, broadcast and multiple access channels. The optimal beam forming vectors are calculated in a iterative manner, i.e. calculating the transmit beam formers for known receive beam formers, and vice versa, calculating receive beam formers for known transmit beam formers, until reaching a globally optimal solution [15].

\section{Material and Methods}

The current trend in mhealth is to use wireless transceivers to interconnect the sensors that monitor the bio-physiological variables, thus forming a WSN. The device may also be required to process and store the collected data and will be referred to as the patient information sink (may be AP, Cloud and Gateway). The MIMO wireless communication between the patients' sensors and the information sink is not restricted to be performed in a single wireless hop. In fact depending on the wireless transceivers range, the use of more than one wireless hop may be required to reach the information sink, it must be sent to the consultant physician may be located in the same campus as the patient or remote location or both.

\section{System model}

Methodology: We consider the general MIMO channel as

$B=H X+n \rightarrow(1)$

Where $n$ is assume as i.i.d zero mean circularly symmetric complex Gaussian with zero mean and unit variance. The capacity maximization of channel can be formulated as

$\max C s(Q)$

$\operatorname{s.tTr}(Q) \leq P s, Q \geq 0 \rightarrow(2)$

where $P S>0$ is the average transmit power.

We can rewrite Equation 2 as

$\max \operatorname{Co}(Q)-t \rightarrow(3)$

s.t $\operatorname{Tr}(Q) \leq P s, C 1(Q) \leq t$

$Q \geq 0, t<0$

However the equation is still non-convex. This kind of nonconvexity can be dealt with Successive Convex Approximation Algorithm (SCA).

\section{Algorithm 1: Iterative precoding algorithm for SCA.}

1: initially set $H, G, P S, Q$, and $i=0$.

2: repeat

3: update $i=i+1$, and compute $D$ with $Q$.

4: compute $Q$

5: compute $C s(Q)$, and update $Q$.

6: until the termination criteria is satisfied. 
7: return $Q$ and $C s$.

\section{Algorithm 2: MASTS in virtual MIMO channel selection}

The MIMO channels with discrete time model may be denoted as

$$
\left[\begin{array}{l}
B 1 \\
B 2 \\
B 3
\end{array}\right]=\left[\begin{array}{ccc}
h 11 & \cdots & h m 1 \\
\vdots & \ddots & \vdots \\
h 1 n & \cdots & h m n
\end{array}\right]\left[\begin{array}{l}
x 1 \\
x 2 \\
x 3
\end{array}\right]+\left[\begin{array}{c}
n 1 \\
n 2 \\
n m
\end{array}\right]
$$

We may write the above equation as $B=H X+n$, where $\mathrm{H}$ is an independent zero mean random matrix and $n$ denotes random noise. We may use a graph of vertices and edges to represent the MIMO environment. To deploy the graph theoretical approach on maximum spanning tree in to MIMO channel selection and program the algorithm.

\section{MASTS algorithm is:}

1) Step 1: Select 3 edges with the largest weight at first (including their vertices).

2) Step 2: Enlarge the sub graph by edges with large weight in decreasing manner and make sure no cycles are formed.

3) Step 3: Continue Step 2 until the edge number of enlarged sub graph is equal to $X_{\mathrm{t}}+X_{\mathrm{r}}-1$. This final sub graph is the spanning tree with the maximum sum of weight. As virtual MIMO graph contains the same information as that of channel gain matrix $\mathrm{H}$, we illustrate MASTS algorithm by matrix entry selection procedure and matrix $H_{\mathrm{b}}, H_{\mathrm{c}}, H_{\mathrm{d}}, H_{\mathrm{e}}$.

\section{Simulation}

In this network system 12 nodes are fixed (Figure 1). These nodes are communicated using 8 antennas. Node 4 is act as a cloud storage Nodes 3, 5 and 9 are act as channel one, two and three respectively. Simultaneously two nodes communicate two different data streams with same frequency. For making a network Initialization takes few seconds to fix the node. It is done by using the AODV protocol.

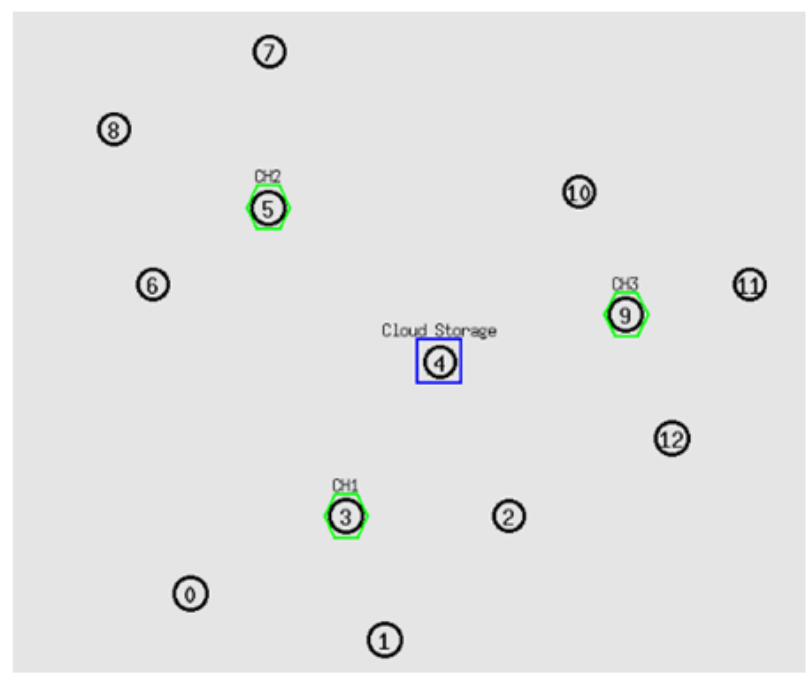

Figure 1. Initializing MIMO nodes.
The number of packets increases in the transmission the latency is decreases and finally the latency is zero. When the packet transmission is from 50 to 100 it maintains the linear property. After that the packets increases the delay reduces consistently. Collision occurs during the transmission between the nodes using MIMO channel, the channels communicate through iteration process and switch over among them. Now, consider an small data transmission from same channel of nodes $\mathrm{A}$ and $\mathrm{B}$, communicates with maximum delay, nodes always waits for an acknowledgement with minimum duration and once fails to acknowledge, then it automatically configured to other channel through iteration process. The large data transmission spitted among the two antennas on the node and communicates concurrently. This is illustrated in Figure 2.

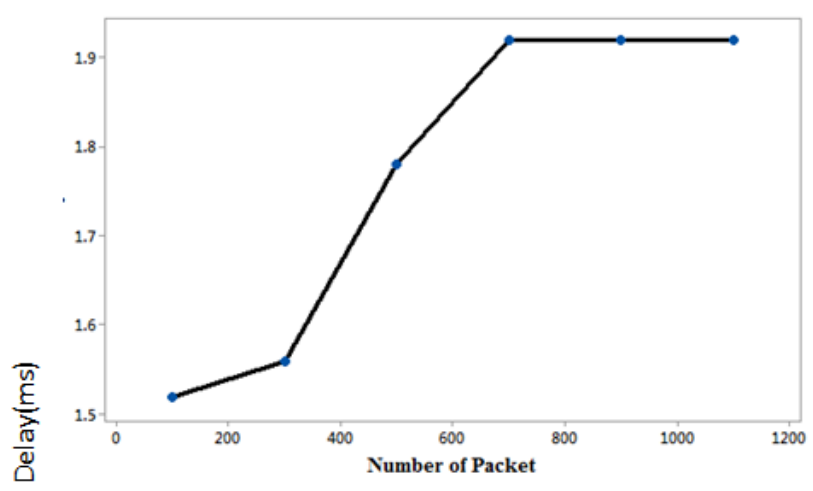

Figure 2. End to end delay.

The packet delivery ratio with time maintains ramp signal between 80 to 88 . Then the packet delivers without delay from transmitter to receiver. Initially the packets are delivered with small latency, then latency reduces slowly and finally when the packet delivery ratio goes above 88 the latency becomes zero from the node. This is analysed in Figure 3.

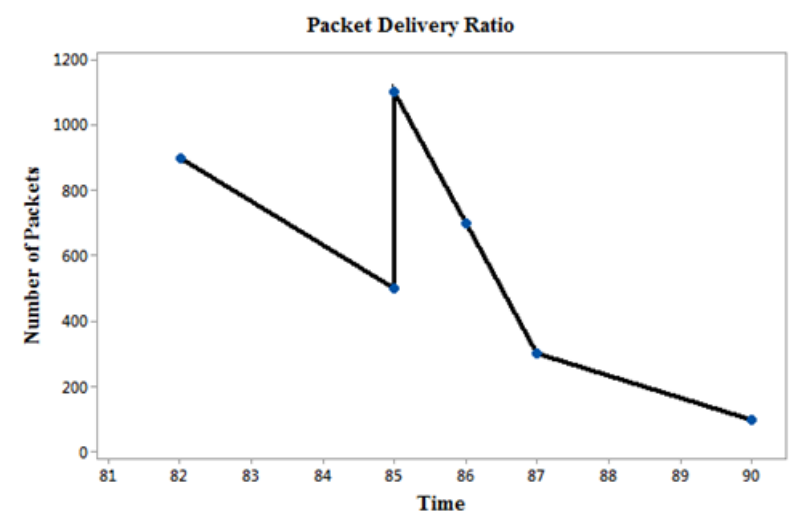

Figure 3. Packet delivery ratio.

The number of packets versus energy maintains ramp signal relationship between them continuously. Finally it maintains the latency is zero. The network energy is sustaining up to the lifetime of service. The residual energy remains available in a network even though the 1000 number of packets transmitted. Initially the capacity of the network is high, it starts reduces after delivery the 900 packets. This is illustrated in Figure 4. 


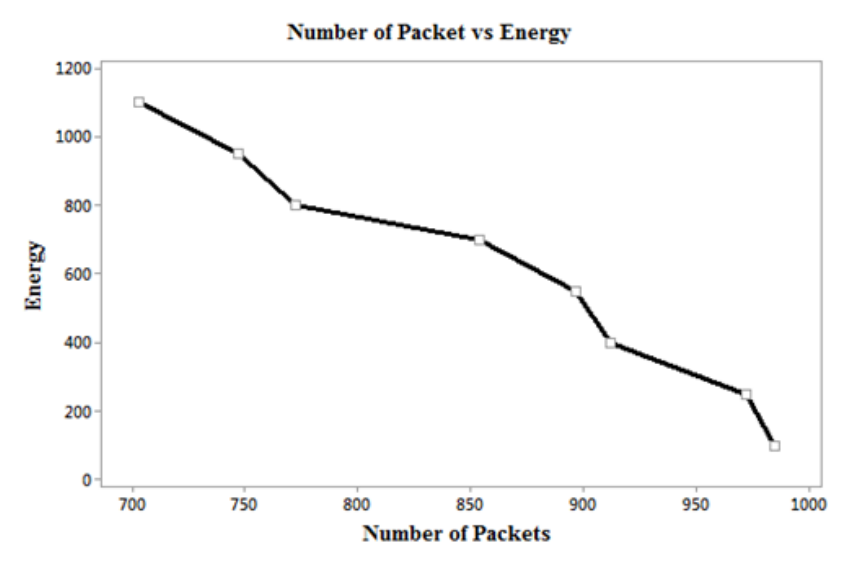

Figure 4. Number of packets versus energy.

Finally the throughput of the network is increases rapidly when the time increases. The transmission data rate is increases with time gradually. This is shown in Figure 5.

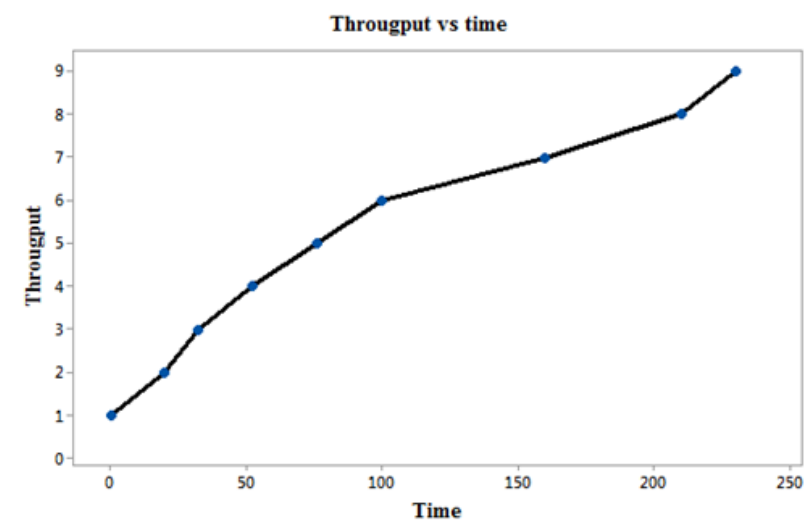

Figure 5. Throughput plot.

\section{Conclusion}

The scheme to address channel selection and energy utility issues associated with MIMO systems. It highlights the merits related with the convexity specifications for the approximate function in WSN for maximization of the energy in the node. The experimental setup with the SCA algorithms improves the battery level of the node and collision of nodes and delays in the transmission is reduced. The Maximum Spanning Tree Searching (MASTS) algorithm to select a set of sub channels that always provide a path connecting all sensors.

\section{Acknowledgement}

I thank A. Vignesh R\&D manager, Chase Technologies., for his suggestions and support.

\section{References}

1. Dragan S, Olga BL, Anders HM, Victor ML. Applications of MIMO techniques to sensing of cardiopulmonary activity. Wireless Communications and Applied Computational Electromagnetics IEE Conference 2005.

2. Chao H, Yang L, Gabriel EA, Thomas PK, Richard DG. In vivo wireless communications and networking. Information Theory and Applications Workshop (ITA) 2015.

3. Anand SK, Keyoor G, Sandeep HK. Increasing wireless channel capacity through mimo systems employing colocated antennas. IEEE Trans Microwav Theor Tech 2005; 53.

4. Aboulnasr H, Sergiy AV. Phased-MIMO radar: a tradeoff between phased-array and MIMO radars. IEEE Trans Sig Proc 2010; 58.

5. Jing L, Qilian L. A graph theoretical algorithm for virtual MIMO channel selection in wireless sensor networks, 2008.

6. Kiranjit K, Sandeep W. Energy efficient wireless sensor networks based on clustering techniques. Int J Comp Appl $2015 ; 119$.

7. Vibhav KS, Richa M, Syed AI. Energy efficient wireless sensor networks using co-operative MIMO: a technical review. Int J Comp Appl 2016; 135.

8. Sharanbasappa S, Parvathi CS. A survey on wireless technologies for biomedical parameters. Int J Sci Res 2013.

9. Bliss DW, Forsy K.W The MIMO radar medical imaging:self-interference mitigation for breast tumor detection. Sig Sys Comp 2006.

10. Sardellitti S, Barbarossa S, Scutari G. Distributed mobile cloud computing. Joint optimization of radio and computational resources Globecom Workshop 2014.

11. Ahmad A. Smart massive MIMO: An infrastructure toward 5th generation smart cities network. Comp Sci Network Intern Architec 2016.

12. Yinsheng L, Geoffrey YL, Wei H, Zhangdui Z. Lowcomplexity recursive convolutional precoding for OFDMbased large-scale antenna systems. IEEE Trans Wireless Commun 2016; 15: 4902-4913.

13. Xiaofei X, Xiang C, Ming Z, Shidong Z, Chong YC, Jing W. Power-efficient distributed beam forming for fullduplex MIMO relaying networks IEEE Trans Vehicul Technol 2016; 10.

14. Radwa S, Lingyang S, Karim GS, Zhu H. Full-duplex meets multiuser MIMO: comparisons and analysis. IEEE Trans Vehicular Technol 2017; 66: 1.

15. Daniel D, Vladimir A, Liljana G, Petri M. Generic multiuser coordinated beam forming for underlay spectrum sharing. IEEE Trans Commun 2016; 64: 2285-2298.

\section{*Correspondence to}

Duraichi N

Anna University

Chennai

India 